Gathering laboratory results to history and clinical examination would improve the sensibility and specificity of ferritinemia for this highly dangerous disease and allow decisive therapeutic decisions.

Conclusion In addition to its "traditional" interest in deficits or iron overload, ferritin may offer some beneficial major diagnostic benefits for life threatening cases like perinatal hemochromatosis.

\section{PO-0289 IT TAKES MORE THAN EIGHT DAYS BEFORE TINZAPARIN LEADS TO ADEQUATE ANTI-XA LEVELS IN PAEDIATRIC INTENSIVE CARE PATIENTS FOLLOWING CONGENITAL HEART SURGERY}

${ }^{1} \mathrm{~A}$ van der Hoeven, ${ }^{1} \mathrm{PP}$ Roeleveld, ${ }^{2} \mathrm{R}$ de Wilde, ${ }^{1} \mathrm{HE}$ Bunker-Wiersma. ${ }^{1}$ Pediatric Intensive Care, Leiden University Medical Center, Leiden, Netherlands; ${ }^{2}$ Intensive Care, Leiden University Medical Center, Leiden, Netherlands

10.1136/archdischild-2014-307384.941

Objective Tinzaparine is used in paediatric intensive care (PICU) following cardiac surgery as a bridge to oral anticoagulation. Low Molecular Weight Heparins (LMWH), such as Tinzaparin are thought to lead to immediate anticoagulation with adequate anti-Xa levels 2-4 hours after the first dose. Dosing following international guidelines, is age dependent and guided by anti-Xa levels. However, little is known about LMWH dosing in PICU patients. We conducted a retrospective study to evaluate tinzaparin dosing.

Methods We retrospectively analysed Tinzaparin doses and antiXa levels from all children admitted to PICU (January 2012December 2013). Hospital policy is to determine the first aXa level after 3-4 doses and $4 \mathrm{~h}$ post dose, targeting $0.5-1.5 \mathrm{IU} / \mathrm{ml}$. Results There were 31 episodes of newly started Tinzaparin in 28 children. Mean age was $57(\mathrm{SD} \pm 62)$ months. First anti-Xa levels were determined at $3.45(\mathrm{SD} \pm 1.9$; range 1-12) days after the first dose and were sub therapeutic in 25 of 31 (81\%): mean 0.33 (SD \pm 0.15 ) IU/ml. Tinzarin dose was increased in $12 / 25$ $(48 \%)$ patients and further anti-Xa levels were determined. In 15 patients further levels were not available due to transition to vit $\mathrm{K}$ antagonists or PICU discharge. Therapeutic anti-Xa levels (0.69 (SD \pm 0.27$) \mathrm{IU} / \mathrm{ml})$, were eventually reached in PICU in 16 patients after a mean of $8.8(\mathrm{SD} \pm 7.1$ range $3-30)$ days.

Conclusion Tinzaparin dosing in PICU patients only leads to target anti-Xa levels after more than 8 days. Levels need to be determined after the first dose so that doses can be adequately increased.

\section{PO-0290 SEIZURES IN A PAEDIATRIC INTENSIVE CARE UNIT: A PROSPECTIVE STUDY}

${ }^{1}$ S Sahin, ${ }^{1} \mathrm{G}$ Ayar, ${ }^{1} \mathrm{M}$ Uysal Yazici, ${ }^{1} \mathrm{~T}$ Koksal, ${ }^{1} \mathrm{R}$ Gunduz, ${ }^{2} \mathrm{Z}$ Karalok, ${ }^{3}$ EBRU Petek Arhan. Pediatric Intensive Care Unit, Ankara Child Health Hematology Oncology Education and Research Hospital, Ankara, Turkey; ${ }^{2}$ Pediatric Neurology Division, Ankara Child Health Hematology Oncology Education and Research Hospital, Ankara, Turkey; ${ }^{3}$ Pediatric Neurology Division, Gazi University School of Medicine, Ankara, Turkey

\subsection{6/archdischild-2014-307384.942}

Purpose To determine the aetiology and clinical features of seizures in critically ill children admitted to paediatric intensive care unit (PICU).

Methods A total of 203 children were admitted from June to November 2013, 45 patients were eligible. Age ranged from 2 months to 19 years. Seizures were organised as epileptic or nonepileptic. Demographic data, PRISM III, GCS, risk factors, coexistent diagnosis, medications administered before admission, type and duration of seizures, drugs used, requirement and duration of mechanical ventilation, length of stay and neuroimaging findings were collected prospectively.

Results The male-female ratio was 0.8. Mean age was 5.4. The most common causes of seizures were non-epileptic. Most frequent coexistent diagnosis was infectious diseases and 53.3\% had recurrent seizures. Medications were administered to $51.1 \%$ of the patients before admission. Seizures were focal in 21 (46.7\%), generalised in $11(24.4 \%)$ and 13 (28.9\%) had status epilepticus. Intravenous midazolam was first line therapy in $48.9 \%$. Non-epileptic seizures were usually new onset and duration was shorter. Epileptic seizures tended to be recurrent and likely to progress to status epilepticus. However, type of seizures didn't change severity of the disease. Also, laboratory test results, medications administered before admission, requirement and duration of ventilation, mortality and length of stay was not significant between epileptic/non-epileptic patients.

Conclusion Seizures in critically ill children that may evolve into status epilepticus is an important condition requiring attention regardless of cause. Intensified educational programs for PICU physicians and international guidelines are necessary for a more efficient approach to children with seizures.

\section{PO-0291 BRAIN DEATH AND ORGAN DONATION OF CHILDREN}

${ }^{1} \mathrm{R}$ Gunduz, 'S Sahin, ${ }^{1} \mathrm{M}$ Uysal Yazici, 'G Ayar, 'H Yakut, 'A Oden Akman, ${ }^{2} \mathrm{H}$ Hirfanoglu, ${ }^{3} \mathrm{G}$ Kalkan. ${ }^{1}$ Pediatric Intensive Care Unit, Ankara Child Health Hematology Oncology Education and Research Hospital, Ankara, Turkey; ${ }^{2}$ Neonatal Intensive Care Unit, Ankara Gazi University School of Medicine, Ankara, Turkey; ${ }^{3}$ Pediatric Intensive Care Unit, Ankara Gazi University School of Medicine, Ankara, Turkey

\subsection{6/archdischild-2014-307384.943}

Objective To define the demographic characteristics, clinical features and outcome of patients with brain death and to emphasise the importance of organ donation in children.

Setting Centre I: 14-bed, tertiary care Paediatric Intensive Care Unit (PICU).

Centre II: Tertiary care Intensive Care Unit (ICU), Trauma centre.

Methods Data were collected from September 2009 to October 2012 retrospectively. Twenty children who were diagnosed as brain death were included. Data including demographics, disease leading to brain death, duration of brain death evaluation, ancillary tests to confirm the brain death, complications and outcome, duration of hospitalisation, status of survival and organ donation were collected for statistical evaluation.

Results The mean age were $6.2 \pm 5.3$ (median:3.8) years. Male/ female ratio was 1.85 . Disease leading to brain death was traumatic brain injury in $11(55 \%)$ patients. The mean duration of brain death evaluation was $6.7 \pm 6.4$ (median:4) and $1.7 \pm 1$ (median:1) days in Centres I and II respectively. The duration of hospitalisation was $12.5 \pm 10.7$ (median:7.5) days. Electroensefalography (EEG) was applied in $18(90 \%)$ patients. Complications included diabetes incipitus in $9(45 \%)$ cases. Duration of survival was $9.8 \pm 9.4$ (median:6) days. One of the patients' parents give consent to organ donation in Centre I while 4 parents accepted organ donation in Centre II. The study demonstrated that duration of brain death evaluation in Centre I was longer when compared to Centre II $(p<0.05)$. There was no difference between centres in obtaining concepts of organ 